\title{
Serum KL-6, CA19-9, CA125 and CEA are Diagnostic Biomarkers for Rheumatoid Arthritis-Associated Interstitial Lung Disease in the Chinese Population
}

\author{
Muhan Zheng $\cdot$ Aiju Lou $\cdot$ Haoru Zhang $\cdot$ Shijie Zhu \\ Min Yang · Weinan Lai
}

Received: October 22, 2020 / Accepted: February 3, 2021 / Published online: February 14, 2021

(C) The Author(s) 2021

\section{ABSTRACT}

Introduction: This study aimed to evaluate the role of tumor marker carbohydrate antigen (CA) 125 (CA125), CA19-9, carcinoembryonic antigen (CEA) and Krebs von den Lungen-6 (KL-6) in the diagnosis and determination of the severity of interstitial lung disease (ILD) in rheumatoid arthritis (RA) patients.

Methods: A retrospective analysis was performed. Fifty RA patients (24 patients with ILD and 26 patients without ILD), 10 healthy subjects and 14 patients with other connective tissue disease-associated interstitial lung disease were included. Serum levels of KL-6 and tumor markers CA19-9, CA125 and CEA were measured. Chest HRCT of patients with ILD was scored quantitatively according to the degree of fibrosis. Data on the C-reactive protein,

M. Zheng · H. Zhang · M. Yang ( $₫) \cdot$ W. Lai ( $₫)$ Department of Rheumatology and Immunology, Nanfang Hospital, Southern Medical University, Guangzhou, Guangdong, China

e-mail: minyang@fimmu.comW. Lai

e-mail: laiweinan@hotmail.com

\section{A. Lou}

Department of Rheumatology and Immunology, Liwan Central Hospital of Guangzhou, Guangzhou, Guangdong, China

\section{S. Zhu}

Department of Cardiology, Nanfang Hospital, Southern Medical University, Guangzhou, Guangdong, China erythrocyte sedimentation rate, rheumatoid factors and anti-cyclic peptide containing citrulline (anti-CCP) were also collected.

Results: Serum levels of KL-6, CA19-9, CA125 and CEA in the RA-ILD group were significantly higher than those in the RA-no-ILD group. The serum KL-6 level was positively correlated with the HRCT fibrosis score $(r=0.63, p=0.002)$. The logistic regression analysis showed that CA19-9 and smoking were associated with RAILD $[\mathrm{OR}=1.118,95 \% \quad \mathrm{CI}=(1.038,1.204)$, $p=0.003$ for CA19-9, OR $=14.969,95 \% \mathrm{CI}=$ (1.750, 128.043), $p=0.013$ for smoking].

Conclusions: KL-6 level and tumor markers were elevated in RA-ILD, and strongly associated with the severity of ILD, supporting their value as pathogenically relevant biomarkers, which can contribute to noninvasive detection of this extra-articular disease complication.

\section{PLAIN LANGUAGE SUMMARY}

Interstitial lung disease (ILD) is a common pulmonary manifestation of RA associated with high morbidity and mortality. Our retrospective study was performed to investigate the clinical utility of tumor marker carbohydrate antigen (CA) 125 (CA125), CA19-9, carcinoembryonic antigen (CEA) and Krebs von den Lungen-6 (KL6) in the diagnosis and determining the severity of RA-ILD. Fifty RA patients (24 patients with 
ILD and 26 patients without ILD), 10 healthy subjects and 14 patients with other connective tissue disease-associated interstitial lung disease (CTD-ILD) were included. The results showed KL-6 level and tumor markers were elevated in RA-ILD, and strongly associated with the severity of ILD, which meant KL-6 and tumor markers might be useful pathogenically relevant biomarkers and could be predictors for the diagnosis and determination of severity of ILD in RA.

Keywords: Interstitial lung disease; KL-6; Rheumatoid arthritis; Tumor markers

\section{Key Summary Points}

Interstitial lung disease (ILD) is a serious complication of rheumatoid arthritis (RA) and is associated with high morbidity and mortality for affected patients

Though ILD is well recognized as the most prevalent comorbidities in RA, less is known about the serum biomarkers of RAILD

Our study showed KL-6 and tumor markers CA19-9, CA125, CEA might be useful predictors for the diagnosis and determination of the severity of ILD in RA

\section{DIGITAL FEATURES}

This article is published with digital features, including a summary slide and plain language summary, to facilitate understanding of the article. To view digital features for this article go to https://doi.org/10.6084/m9.figshare. 13626398.

\section{INRODUCTION}

Rheumatoid arthritis (RA) is a common systemic autoimmune and inflammatory disease associated with progressive impairment, systemic complications and increased mortality [1]. Approximately $40 \%$ of RA patients may present with the extra-articular manifestations, and pulmonary involvement is a common feature [2]. Interstitial lung disease (ILD) is the primary pulmonary manifestation of RA, which can be detected in $60 \%$ of RA patients on highresolution computed tomography (HRCT), and is a leading cause of substantial morbidity and mortality for affected patients [3, 4]. The median survival for patients with RA overall was reported to be 9.9 years, while median survival after ILD diagnosis was 2.6 years [5]. However, Solomon et al. found overall mortality rates in RA are declining, and the rate of death due to rheumatoid arthritis-associated interstitial lung disease (RA-ILD) has increased significantly [6].

To manage RA-ILD, early diagnosis and monitoring of disease progression are important. At present, the diagnosis of RA-ILD mainly depends on HRCT, but costs and ionizing radiation may limit its use in clinical practice, and lung tissue itself is relatively inaccessible. Finding new non-invasive markers associated with RA-ILD is urgently needed.

Tumor markers were mainly referred to biological substances synthesized and released by tumor cells or other cells in response to tumor tissue, which can play a certain role in the clinical diagnosis of related tumors. The commonly used clinical tumor markers were carbohydrate antigen (CA) 125 (CA125) and CA19-9, carcinoembryonic antigen (CEA). In recent years, tumor markers have been found to be associated with ILD in connective tissue disease (CTD) [7]. Shi et al. observed that higher serum CA153 and neuron-specific enolase (NSE) levels are significantly related to increased risk of ILD in patients with primary Sjögren's syndrome (pSS) rather than the EULAR Sjögren's syndrome disease activity index (ESSDAI) [8]. Elevated CA125 was associated with a sixfold increase in the odds of ILD in RA [9].

Krebs von den Lungen-6 (KL-6) is a mucinlike glycoprotein, which has profibrotic and anti-apoptotic effects on lung fibroblasts. KL-6 is considered to reflect the degree and severity of alveolar epithelial injury and might be a useful biomarker for predicting the prognosis of 
idiopathic pulmonary fibrosis (IPF) [10-12]. In recent years, numerous studies have focused on the relationship between KL- 6 and ILD $[13,14]$. Lee et al. found KL-6 levels were higher in RAILD than RA-no-ILD patients [15]. However, whether KL-6 can distinguish ILD in patients with RA and be a biomarker for the presence of RA-ILD is still unknown; the relationship between KL- 6 and tumor markers in RA-ILD has also not been reported.

The assessment of tumor markers and KL- 6 be of clinical significance for identifying and monitoring RA-ILD. Based on these considerations, the current study aimed to investigate their role in the diagnosis and determination of the severity of RA-ILD.

\section{METHODS}

\section{Study Design and Patients}

This is a retrospective analysis of 50 patients who met the 1987 American College of Rheumatology (ACR) criteria for definite RA [16] at Nanfang Hospital of Southern Medical University between January 2019 and September 2019. Patients were excluded from this study if they were suffering from other pulmonary diseases, such as pulmonary infection, pulmonary tuberculosis, chronic obstructive pulmonary disease and ILD caused by pneumoconiosis; inhalation of organic matter and other causes; sarcoidosis and amyloidosis; a history of malignancy or other disease that might affect levels of tumor markers; or a recent clinically significant infection (HIV, viral hepatitis, etc.). Ten healthy controls (HC) matched by age and gender were also enrolled. An internal reference group was also included, which was composed of 14 other patients with CTD-ILD (including 3 patients with mixed connective tissue disease, 3 patients with scleroderma, 2 patients with dermatomyositis, 3 patient with myositis, 1 patient with Sjogren's syndrome, 1 patient with Raynaud syndrome and 1 patient with anti-synthase antibody syndrome), which met the classification criteria of corresponding CTD [17-19]. All enrolled subjects underwent chest HRCT scans, and HRCT was evaluated by two radiologists and one respiratory surgeon, who were blinded. The diagnosis and evaluation of interstitial lung disease was made according to chest HRCT [20]. All procedures performed in studies involving human participants were in accordance with the ethical standards of the institutional and/or national research committee and with the 1964 Helsinki declaration and its later amendmentsor comparable ethical standards. The study was approved by the Ethics Committee of Nanfang Hospital, Southern Medical University (NFEC-2019-240). Informed consent was obtained from all individual participants included in the study.

\section{HRCT Fibrosis Scoring}

Fibrosis was scored using an arbitrary semiquantitative scale from 0 to $\mathrm{V}$ [21]: grade 0 ( 0 points): no fibrosis; grade 1 (1 point), presence of abnormal lobular structure, subpleural vertical line shadow, subpleural arc shadow, etc.; grade 2 ( 2 points): abnormal lobular structure, abnormal interstitial shadow around the bronchovascular bundle, thickening of the bronchovascular bundle, deformation of the lobular outline, interlobular septum, etc.; grade 3 (3 points): lobular morphological abnormality with abnormal interstitial shadow around the bronchovascular bundle and $<10 \mathrm{~mm}$ honeycomb shadow; grade 4 (4 points): lobular morphological abnormality with abnormal interstitial shadow around the bronchovascular bundle and the extent of the abnormal interstitial shadow is $10-30 \mathrm{~mm}$; grade 5 (5 points): abnormal interstitial shadow around the bronchioles and vascular bundles and $>30 \mathrm{~mm}$ honeycomb shadow; the lung texture is unclear or has disappeared. The two lungs are artificially divided into six lung areas: the left upper lung, left middle lung, left lower lung, right upper lung, right middle lung area and right lower lung area. The upper aortic arch was taken as the upper lung area, below the aortic arch to the right inferior pulmonary vein as the middle lung area, and below the right inferior pulmonary vein as the lower lung area. HRCT fibrosis score of patients with ILD was scored 
blindly by two imaging doctors and one respiratory doctor. Finally, the average value was calculated as the final score. The severity of pulmonary interstitial fibrosis was determined according to the score.

\section{Clinic and Laboratory Indexes}

Clinical data were collected from patient records using electronic data processing. The data collected included demographic information and systemic inflammation [C-reactive protein (CRP), erythrocyte sedimentation rate (ESR)]. Plasma levels of rheumatoid factors (RF) and anti-cyclic peptide containing citrulline (anti-CCP) were also recorded.

\section{Circulating Biomarkers}

All serum samples were stored at $-80{ }^{\circ} \mathrm{C}$ prior to use. Tumor markers including CEA, CA125 and CA19-9 were measured in the laboratory at Nanfang Hospital. The concentration of CA125 was determined by chemiluminescent microparticle immunoassay. The concentrations of CA19-9 and CEA were measured by the Bayer Centaur automatic chemiluminescence immunoassay system. The concentration of KL6 was measured by chemiluminescence immunoassay with the LumipulseG1200 kit according to the manufacturer's instructions. The normal ranges of each tumor-associated antigen (TAA) are defined as follows: CA19$9<37.00 \mathrm{U} / \mathrm{ml} ; \mathrm{CA} 125<35.00 \mathrm{U} / \mathrm{ml} ; \mathrm{CEA}<5$ $\mu \mathrm{g} / \mathrm{l}$.

\section{Statistical Analysis}

Continuous variables are summarized as median [interquartile ranges (IQR)] or mean $\pm \mathrm{SD}$ and categorical data with frequency (percentages). The clinical characteristics and serological markers of different groups were compared using Student's $t$-test or Mann-Whitney $U$ test (for continuous data) or chi-squared test (for categorical data). We used Spearman's correlation coefficient by rank to analyze the correlation between serological markers. Binary logistic regression analysis was used to analyze the risk factors of interstitial lung disease in patients with RA. The receiver-operating characteristic (ROC) curve was used to analyze the value of each serological marker in the diagnosis of RA-ILD. SPSS 20.0 (SPSS Inc., Chicago, IL, USA) was used to complete the analysis. $P<0.05$ was considered statistically significant.

\section{RESULTS}

\section{Characteristics of the RA Patients}

Of 50 RA patients, 24 were identified as RA-ILD and 26 as RA-no-ILD cases based on evidence of HRCT imaging scans. Clinical features of the included patients are shown in Table 1 . The mean age of RA-ILD patients was $62.9 \pm 11.8$ years and that of RA-non-ILD cases was $56.5 \pm 10.3$ years. Percentages of males and smokers were higher in RA-ILD than in RA-noILD patients. RA-ILD patients were more likely to be older and had higher RF levels than RAno-ILD patients, but this association was not statistically different between the two groups. RA-ILD patients had higher CRP levels than RAnon-ILD patients $(p<0.05)$, while other general biological indexes, such as the duration of the disease process, anti-CCP, RF and ESR, showed no significant difference between the two groups.

\section{Association Between Serum Tumor Markers, KL-6 and Disease Activity Indicators}

We compared the tumor markers and KL-6 level among the different groups; the results showed the levels of KL-6, CA19-9, CA125 and CEA in the RA-ILD group were apparently increased compared to the RA-no-ILD group, and the CA19-9 level in the RA-ILD group was also significantly higher than in the CTD-ILD group, while tumor markers and KL-6 showed no significant difference between the RA-no-ILD and HC patients. The HRCT score and KL-6 level were comparable between the CTD-ILD and RAILD patients (Table 1 and Fig. 1). 
Table 1 Demographic and clinical features of patients at baseline

\begin{tabular}{|c|c|c|c|c|c|c|c|c|c|}
\hline & $\begin{array}{l}\text { RA-no- } \\
\text { ILD } \\
N=26\end{array}$ & $\begin{array}{l}\text { RA-ILD } \\
N=24\end{array}$ & $\begin{array}{l}\mathrm{HC} \\
N=10\end{array}$ & $\begin{array}{l}\text { CTD-ILD } \\
N=14\end{array}$ & $\begin{array}{l}\text { RA-ILD } \\
\text { vs. RA- } \\
\text { no-ILD }\end{array}$ & $\begin{array}{l}\text { RA- } \\
\text { ILD vs. } \\
\text { HC }\end{array}$ & $\begin{array}{l}\text { RA-ILD } \\
\text { vs. } \\
\text { CTD- } \\
\text { ILD }\end{array}$ & $\begin{array}{l}\text { RA-no- } \\
\text { ILD vs. } \\
\text { HC }\end{array}$ & $\begin{array}{l}\text { CTD- } \\
\text { ILD } \\
\text { vs. HC }\end{array}$ \\
\hline Age, years & $56.6 \pm 10.3$ & $62.9 \pm 11.8$ & $52.2 \pm 6.4$ & $53.1 \pm 5.9$ & 0.051 & 0.011 & 0.002 & 0.218 & 0.734 \\
\hline Male sex & $6(23.1)$ & $14(58.3)$ & $2(20.0)$ & $5(35.7)$ & 0.012 & 0.063 & 0.313 & 1.000 & 0.653 \\
\hline Ever-smoker & $4(15.4)$ & $12(50.0)$ & $1(10.0)$ & $3(21.4)$ & 0.015 & 0.051 & 0.101 & 1.000 & 0.615 \\
\hline BMI, $\mathrm{kg} / \mathrm{m}^{2}$ & $22.2 \pm 4.0$ & $23.1 \pm 4.1$ & $25.8 \pm 3.8$ & $22.6 \pm 3.2$ & 0.613 & 0.112 & 0.917 & 0.024 & 0.041 \\
\hline $\begin{array}{l}\text { RA } \\
\text { duration, } \\
\text { years }\end{array}$ & $3(0.2,30)$ & $\begin{array}{c}4.5(0.6 \\
26.0)\end{array}$ & & & 0.427 & & & & \\
\hline $\mathrm{RF}, \mathrm{IU} / \mathrm{ml}$ & $\begin{array}{c}305.6(9.5 \\
3560.0)\end{array}$ & $\begin{array}{c}561.8(65.3 \\
2730)\end{array}$ & & & 0.054 & & & & \\
\hline $\begin{array}{l}\text { Anti- } \\
\text { CCP,RU/ } \\
\text { ml }\end{array}$ & $\begin{array}{c}175.6(0.5 \\
1200.0)\end{array}$ & $\begin{array}{c}205.0(0.5 \\
1200.0)\end{array}$ & & & 0.651 & & & & \\
\hline CRP, mg/l & $\begin{array}{r}6.4(0.3 \\
135.4)\end{array}$ & $\begin{array}{c}54.5(0.4 \\
246.1)\end{array}$ & $\begin{array}{c}1.8(0.2 \\
11.8)\end{array}$ & $\begin{array}{c}11.7(0.2 \\
90.9)\end{array}$ & 0.003 & $<0.001$ & 0.032 & 0.012 & 0.051 \\
\hline $\mathrm{ESR}, \mathrm{mm} / \mathrm{h}$ & $\begin{array}{c}60.5(2.0 \\
132.0)\end{array}$ & $\begin{array}{c}54.5(13 \\
125.0)\end{array}$ & & & 0.834 & & & & \\
\hline $\mathrm{KL}-6, \mathrm{U} / \mathrm{ml}$ & $\begin{array}{c}227.0 \\
(136.0 \\
581.0)\end{array}$ & $\begin{array}{l}558.0 \\
\quad(221.0, \\
3575.0)\end{array}$ & $\begin{array}{c}232.0 \\
(179.0 \\
777.0)\end{array}$ & $\begin{array}{l}824.5 \\
\quad(348.0, \\
3866.0)\end{array}$ & $<0.001$ & 0.001 & 0.365 & 0.489 & 0.001 \\
\hline $\begin{array}{r}\text { CA125, } \\
\mathrm{U} / \mathrm{ml}\end{array}$ & $\begin{array}{c}9.0(3.5 \\
17.8)\end{array}$ & $\begin{array}{c}40.0(7.1 \\
75.0)\end{array}$ & $\begin{array}{c}8.3(4.3 \\
16.8)\end{array}$ & $\begin{array}{c}16.2(5.9 \\
171.3)\end{array}$ & $<0.001$ & $<0.001$ & 0.098 & 0.453 & 0.016 \\
\hline $\begin{array}{c}\text { CA19-9, } \\
\mathrm{U} / \mathrm{ml}\end{array}$ & $\begin{array}{c}16.2(6.7 \\
42.8)\end{array}$ & $\begin{array}{c}56.8(11.8 \\
521.6)\end{array}$ & $\begin{array}{c}12.1(2.4 \\
29.9)\end{array}$ & $\begin{array}{c}13.7(4.4 \\
100.4)\end{array}$ & $<0.001$ & $<0.001$ & $<0.001$ & 0.258 & 0.482 \\
\hline $\mathrm{CEA}, \mu \mathrm{g} / 1$ & $\begin{array}{c}0.9(0.1 \\
2.8)\end{array}$ & $\begin{array}{c}1.6(0.5 \\
3.8)\end{array}$ & $\begin{array}{c}0.8(0.1 \\
2.6)\end{array}$ & $\begin{array}{c}0.9(0.1 \\
3.5)\end{array}$ & 0.009 & 0.050 & 0.330 & 0.929 & 0.342 \\
\hline $\begin{array}{l}\text { HRCT } \\
\text { fibrosis } \\
\text { score }\end{array}$ & & $14.6 \pm 7.3$ & & $18.3 \pm 5.9$ & & & 0.121 & & \\
\hline
\end{tabular}

Data are presented as mean $\pm \mathrm{SD}$

RA-no-ILD rheumatoid arthritis-without interstitial lung disease, $R A-I L D$ rheumatoid arthritis-related interstitial lung disease, $H C$ healthy controls, $C T D-I L D$ connective tissue disease-associated interstitial lung disease, $B M I$ body mass index, $R F$ rheumatoid factor, Anti-CCP anti-cyclic peptide containing citrulline, CRP c-reactive protein, ESR erythrocyte sedimentation rate, KL-6 Krebs von den Lungen-6, CA125 carbohydrate antigen 125, CA19-9 carbohydrate antigen 19-9, CEA carcinoembryonic antigen, HRCT high-resolution computed tomography 

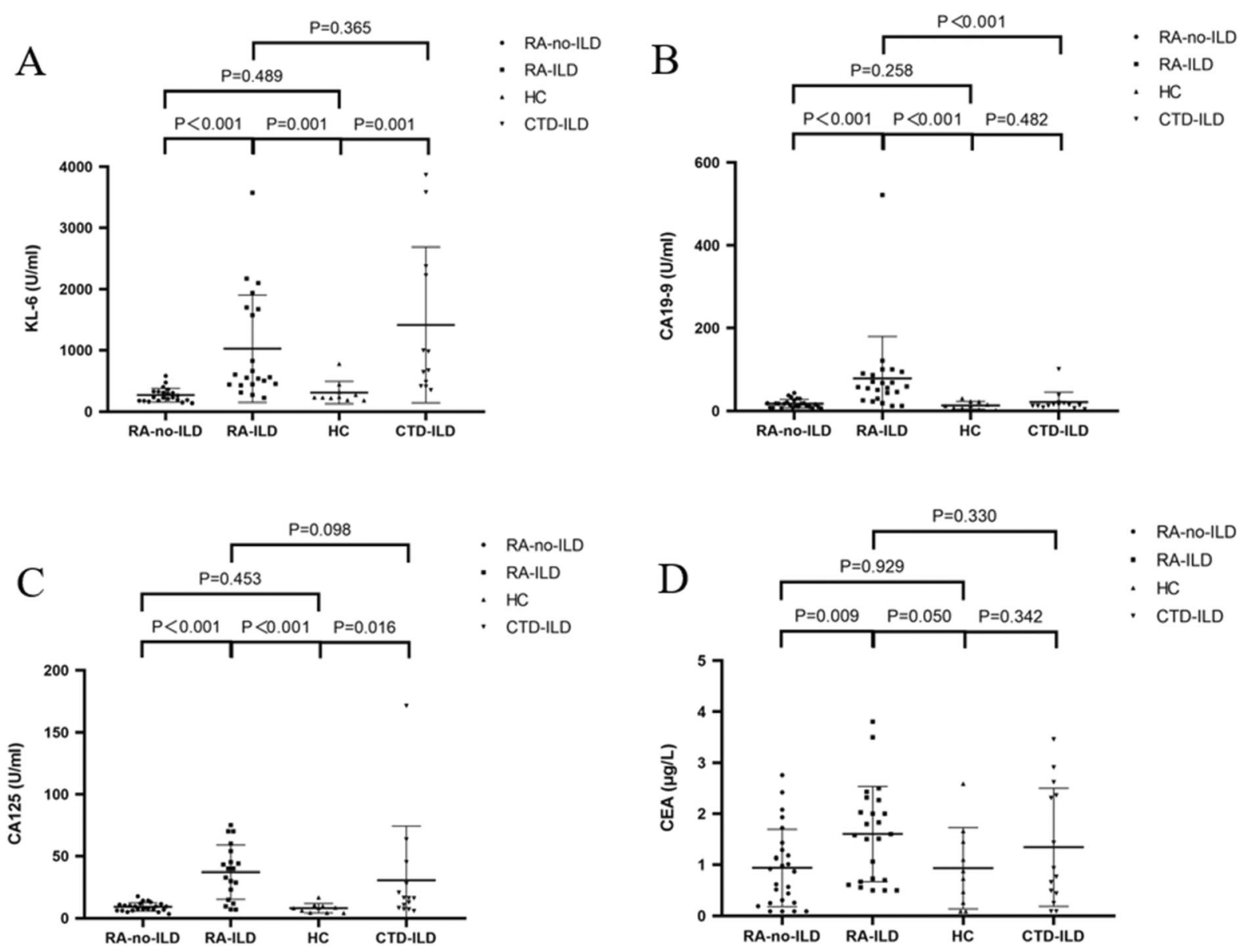

Fig. 1 Elevated KL-6 and tumor markers level in RA-ILD patients. The levels of KL-6 (a), CA19-9 (b), CA125 (c) and CEA (d) were significantly higher in RA-ILD patients. RA-no-ILD rheumatoid arthritis-without interstitial lung disease, $R A-I L D$ rheumatoid arthritis-related

We also explored the correlation between these potential new biomarkers and the severity of ILD by Spearman analysis and found the KL- 6 level was apparently correlated with the HRCT fibrosis score in the RA-ILD group and CTD-ILD group. No obvious correlation was found between the tumor markers and HRCT fibrosis score in any of the groups (Table 2).

We next evaluated the strength of correlation between RA-ILD and tumor markers as well as KL- 6 by using logistic regression analysis. KL6, CA125, CA19-9, CEA, CRP, RF, age, sex and smoking history were included in this model. After adjusting the mutual influence of factors, the serum levels of CA19-9 [odds ratio $(\mathrm{OR})=$

interstitial lung disease, $H C$ healthy controls, CTD-ILD connective tissue disease-associated interstitial lung disease, KL-6 Krebs von den Lungen-6, CA125 carbohydrate antigen 125, CA19-9 carbohydrate antigen 19-9, CEA carcinoembryonic antigen

$1.118,95 \%$ confidence interval $(\mathrm{CI})=(1.038$, 1.20)] and smoking $[\mathrm{OR}=14.969,95 \% \mathrm{CI}=$ $(1.750,128.043)]$ were significantly associated with RA-ILD, which meant high CA19-9 levels and smoking may increase the morbidity of ILD in RA (Table 3).

\section{Clinical Values of Tumor Markers and KL- 6 in the Diagnosis of ILD in RA Patients}

An ROC assessment was performed to evaluate the diagnostic value of the studied tumor markers and KL-6. As Fig. 2 shows, high levels of tumor markers and KL-6 are closely related to 
Table 2 Correlation of the severity of ILD in RA with KL-6 and tumor markers

\begin{tabular}{llllll}
\hline & \multicolumn{2}{l}{ HRCT fibrosis score of RA-ILD } & & \multicolumn{2}{l}{ HRCT fibrosis score of CTD-ILD } \\
\cline { 2 - 3 } & $\boldsymbol{r}$ & $\boldsymbol{p}$ & & $\boldsymbol{r}$ & $\boldsymbol{p}$ \\
\hline KL-6 & 0.63 & 0.00 & 0.69 & 0.009 \\
CA125 & 0.25 & 0.31 & & 0.51 & 0.051 \\
CA19-9 & 0.28 & 0.19 & & 0.41 & 0.13 \\
CEA & 0.33 & 0.12 & 0.45 & 0.087 \\
\hline
\end{tabular}

KL-6 Krebs von den Lungen-6, CA125 carbohydrate antigen 125, CA19-9 carbohydrate antigen 19-9, CEA carcinoembryonic antigen, $H R C T$ high-resolution computed tomography, RA-ILD rheumatoid arthritis-related interstitial lung disease, $H C$ healthy controls, $C T D-I L D$ connective tissue disease-associated interstitial lung disease

Table 3 Logistic regression analysis of the association of RA-ILD and tumor markers

\begin{tabular}{llll}
\hline Variate & OR & 95\% CI & $p$ \\
\hline Smoking & 14.969 & $1.750-128.043$ & 0.013 \\
CA19-9 & 1.118 & $1.038-1.204$ & 0.003 \\
\hline
\end{tabular}

RA-ILD rheumatoid arthritis-related interstitial lung disease, CA19-9 carbohydrate antigen 19-9, CI confidence interval, $O R$ odds ratio

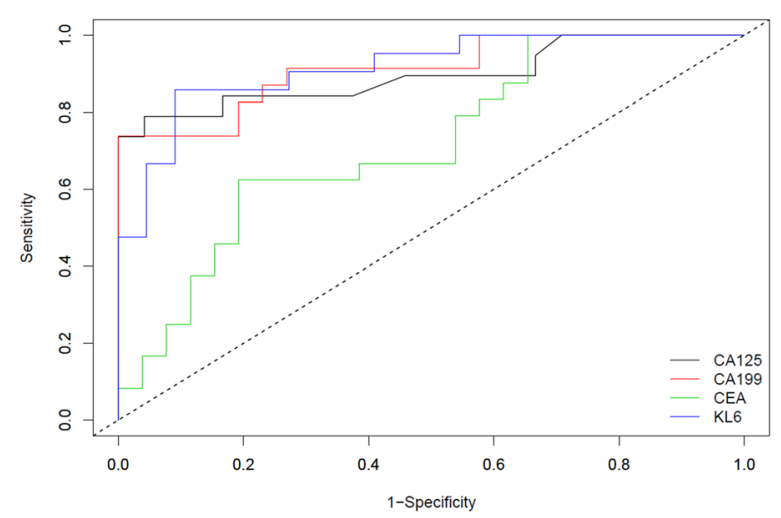

Fig. 2 Predictive capacity of tumor markers and KL-6 in the presence of ILD in RA. KL-6 Krebs von den Lungen6, CA125 carbohydrate antigen 125, CA19-9 carbohydrate antigen 19-9, CEA carcinoembryonic antigen, $R A-I L D$ rheumatoid arthritis-related interstitial lung disease

RA-ILD. The area under the ROC curve (AUC) of all four markers are $>0.7$, i.e, KL-6 (AUC $=$ $0.916,95 \%$ CI $=0.790-0.978)$, CA125 $($ AUC $=$
0.896, 95\% CI $=0.764-0.968)$, CA19-9 $($ AUC $=$ $0.911, \quad 95 \% \quad \mathrm{CI}=0.795-0.974)$ and $\mathrm{CEA}$ $(\mathrm{AUC}=0.716, \quad 95 \% \quad \mathrm{CI}=0.571-0.835) . \quad$ The sensitivity, specificity and best critical value were shown in Table 4.

\section{DISCUSSION}

ILD is a common pulmonary manifestation of RA associated with high morbidity and mortality [22], but the diagnostic value of biomarkers of RA-ILD is still unkown. In our study, the levels of serum CEA, CA19-9, CA125 and KL-6 in RA-ILD were significantly higher than those in RA-no-ILD, which was consistent with previous reports $[14,23]$. These markers were comparable between RA-no-ILD and HC, suggesting that tumor markers and KL-6 may have specific correlations with ILD but not RA, which means RA patients with a high level of KL- 6 and tumor markers may be more likely to develop ILD. The ROC curve further revealed that the circulating KL-6 and tumor markers were positive biomarkers for RA-ILD.

Tumor markers are measurable biochemicals in body tissues, fluid and feces and play an important role in the prognosis and severity of malignancy [24, 25]. In addition to cancer, many other diseases also can lead to elevated tumor markers. A large multicenter sample study found that CA125 and CA19-9 were biomarkers of epithelial injury in patients with IPF [26]. It was also reported that the expression of CA19-9 in ILD with CTD was increased and 
Table 4 Sensitivity, specificity, positive predictive value (PPV), negative predictive value (NPV), area under the ROC curve (AUC) and accuracy of cut-off values in RA-ILD

\begin{tabular}{lllllllll}
\hline Variable & Suggested cut-off & Sensitivity & Specificity & AUC & 95\% CI & Youden's index & PPV & NPV \\
\hline KL-6 & 399 & $85.71 \%$ & $90.91 \%$ & 0.916 & {$[0.790-0.978]$} & 0.7662 & $85.7 \%$ & $86.4 \%$ \\
CA19-9 & 42.83 & $73.91 \%$ & $100.00 \%$ & 0.911 & {$[0.795-0.974]$} & 0.7391 & $94.4 \%$ & $80.6 \%$ \\
CEA & 1.43 & $62.50 \%$ & $80.77 \%$ & 0.716 & {$[0.571-0.835]$} & 0.4327 & $71.4 \%$ & $69.0 \%$ \\
CA125 & 14.3 & $78.95 \%$ & $95.83 \%$ & 0.896 & {$[0.764-0.968]$} & 0.7478 & $88.2 \%$ & $84.6 \%$ \\
\hline
\end{tabular}

KL-6 Krebs von den Lungen-6, CA125 carbohydrate antigen 125, CA19-9 carbohydrate antigen 19-9, CEA carcinoembryonic antigen, $P P V$ positive predictive value, $N P V$ negative predictive value, $R O C$ relative operating characteristic curve, $C I$ confidence interval, $A U C$ area under the ROC curve

the level of CA19-9 was inversely related to the diffusion capacity of the lung for carbon monoxide (DLCO). Moreover, after treatment with immunosuppressants, the CA19-9 level in CTD-ILD was decreased [27]. In our research, tumor markers CA125, CA19-9 and CEA were increased in RA-ILD and might play a role in the occurrence and development of ILD, which means these three tumor markers are not only risk factors for cancer, but also diagnostic indicators for RA-ILD. The reason for the elevated tumor marker levels is not clear yet. The main pathogenesis of ILD might be the sustained injury, over repair, apoptosis of epithelial cells and formation of fibroblast foci. CA125 and CEA have been reported to reflect the proliferation and secretion of epithelial cells, which may provide new thoughts about the correlation between tumor markers and ILD [28]. Although the diagnostic value of tumor markers was obtained by the ROC curve, its specificity and sensitivity in clinical application need to be further observed, and the underlying mechanism is still unknown.

KL-6 is a chemotactic factor of lung fibroblasts and considered to be a marker for the degree and activity of inflammation as well as pulmonary fibrosis [29]. Serum KL-6 is elevated in $70-100 \%$ of patients with various ILDs, such as IPF, CTD-ILD and hypersensitivity pneumonia [30]. The use of drugs may affect serum KL-6 levels in RA patients. In a retrospective study of RA, the KL-6 level was obviously higher in patients treated with biological agents than in those treated with methotrexate [31]. Our results confirmed the level of KL-6 was significantly increased in both RA-ILD and CTD-ILD patients; further analysis found KL-6 had positive correlation with the HRCT fibrosis score, which meant a high KL-6 level was an important discriminating factor of ILD, and it might be a useful predictor for the severity of ILD in RA patients.

Our study also has several limitations. First, since this was a retrospective study, we were unable to follow up the occurrence of cancer and mortality in all enrolled patients in the following years. Second, the number of patients included in this study was relatively small, which limited our power to explore the statistical differences in different lung imaging patterns. Finally, detailed treatment information such as the type, dose, timing and duration of medication was not included in the analysis of prognostic factors. Further studies with more patients, lung biopsy, pulmonary function and/ or BALF testing are needed to explore the actual relationships between RA-ILD and tumor markers, KL-6 and the possible mechanisms. All these important limitations should be addressed in subsequent prospective studies.

\section{CONCLUSIONS}

In conclusion, this study aimed to identify new non-invasive markers of RA-ILD, which can facilitate earlier diagnosis and treatment of this disease. The results demonstrated a strong correlation of circulating tumor marker CA125, 
CA19-9, CEA and KL-6 with the onset and disease severity of ILD in RA patients, which meant these may hold promise as useful biomarkers for RA-ILD.

\section{ACKNOWLEDGEMENTS}

We express our thanks to all the participants for their contributions to this study. We also thank Hao Ren, Qin Huang and Meida Fan for their assitance.

Funding. This study was supported by the Natural Science Foundation of Guangdong Province (grant no. 2017A030313508). All authors had full access to all the data in this study and take full responsibility for the integrity and accuracy of the data analysis. The Rapid Service Fee was funded by the authors.

Authorship. All named authors meet the International Committee of Medical Journal Editors (ICMJE) criteria for authorship for this article, take responsibility for the integrity of the work as a whole and have given their approval for this version to be published.

Authorship Contributions. Muhan Zheng and Aiju Lou contributed equally to this work.

Disclosures. Muhan Zheng, Aiju Lou, Shijie Zhu, Jinjun Zhao, Min Yang and Weinan Lai have nothing to disclose.

Compliance with Ethics Guidelines. All procedures performed in studies involving human participants were in accordance with the ethical standards of the institutional and/or national research committee and with the 1964 Helsinki Declaration and its later amendments or comparable ethical standards. The study was approved by the Ethics Committee of Nanfang Hospital, Southern Medical University (NFEC2019-240). Informed consent was obtained from all individual participants included in the study.

Data Availability. The datasets generated during and/or analyzed during the current study are available from the corresponding author on reasonable request.

Open Access. This article is licensed under a Creative Commons Attribution-NonCommercial 4.0 International License, which permits any non-commercial use, sharing, adaptation, distribution and reproduction in any medium or format, as long as you give appropriate credit to the original author(s) and the source, provide a link to the Creative Commons licence, and indicate if changes were made. The images or other third party material in this article are included in the article's Creative Commons licence, unless indicated otherwise in a credit line to the material. If material is not included in the article's Creative Commons licence and your intended use is not permitted by statutory regulation or exceeds the permitted use, you will need to obtain permission directly from the copyright holder. To view a copy of this licence, visit http://creativecommons.org/licenses/by$\mathrm{nc} / 4.0 /$.

\section{REFERENCES}

1. McInnes IB, Schett G. The pathogenesis of rheumatoid arthritis. N Engl J Med. 2011;365(23): 2205-19.

2. Koduri G, Norton S, Young A, Cox N, Davies P, Devlin J, Dixey J, Gough A, Prouse P, Winfield J, Williams P. Interstitial lung disease has a poor prognosis in rheumatoid arthritis: results from an inception cohort. Rheumatology (Oxford). 2010;49(8):1483-9.

3. Raimundo K, Solomon JJ, Olson AL, Kong AM, Cole AL, Fischer A, Swigris JJ. Rheumatoid arthritis-interstitial lung disease in the United States: prevalence, incidence, and healthcare costs and mortality. J Rheumatol. 2019;46(4):360-9.

4. Hyldgaard C, Hilberg O, Pedersen AB, Ulrichsen SP, Lokke A, Bendstrup E, Ellingsen T. A populationbased cohort study of rheumatoid arthritis-associated interstitial lung disease: comorbidity and mortality. Ann Rheum Dis. 2017;76(10):1700-6.

5. Bongartz $T$, Nannini C, Medina-Velasquez YF, Achenbach SJ, Crowson CS, Ryu JH, Vassallo R, Gabriel SE, Matteson EL. Incidence and mortality of interstitial lung disease in rheumatoid arthritis: a 
population-based study. Arthritis Rheum. 2010;62(6):1583-91.

6. Olson AL, Swigris JJ, Sprunger DB, Fischer A, Fernandez-Perez ER, Solomon J, Murphy J, Cohen M, Raghu G, Brown KK. Rheumatoid arthritis-interstitial lung disease-associated mortality. Am J Respir Crit Care Med. 2011;183(3):372-8.

7. De Luca G, Bosello SL, Berardi G, Rucco M, Canestrari G, Correra M, Mirone L, Forni F, Di Mario C, Danza FM, Pirronti T, Ferraccioli G. Tumour-associated antigens in systemic sclerosis patients with interstitial lung disease: association with lung involvement and cancer risk. Rheumatology (Oxford). 2015;54(11):1991-9.

8. Shi L, Han XL, Guo HX, Wang J, Tang YP, Gao C, Li $\mathrm{XF}$. Increases in tumor markers are associated with primary Sjogren's syndrome-associated interstitial lung disease. Ther Adv Chronic Dis. 2020;11: 2040622320944802.

9. Sargin G, Kose R, Senturk T. Tumor-associated antigens in rheumatoid arthritis interstitial lung disease or malignancy? Arch Rheumatol. 2018;33(4):431-7.

10. Yokoyama A, Kohno N, Hamada H, Sakatani M, Ueda E, Kondo K, Hirasawa Y, Hiwada K. Circulating KL- 6 predicts the outcome of rapidly progressive idiopathic pulmonary fibrosis. Am J Respir Crit Care Med. 1998;158(5 Pt 1):1680-4.

11. Hamai $\mathrm{K}$, Iwamoto $\mathrm{H}$, Ishikawa $\mathrm{N}$, Horimasu $\mathrm{Y}$, Masuda T, Miyamoto S, Nakashima T, Ohshimo S, Fujitaka K, Hamada H, Hattori N, Kohno N. Comparative study of circulating MMP-7, CCL18, KL-6, SP-A, and SP-D as disease markers of idiopathic pulmonary fibrosis. Dis Mark. 2016;2016:4759040.

12. Sokai A, Tanizawa K, Handa T, Kanatani K, Kubo T, Ikezoe K, Nakatsuka Y, Tokuda S, Oga T, Hirai T, Nagai S, Chin K, Mishima M. Importance of serial changes in biomarkers in idiopathic pulmonary fibrosis. ERJ Open Res. 2017;3(3):00019-2016.

13. Kim HC, Choi KH, Jacob J, Song JW. Prognostic role of blood KL-6 in rheumatoid arthritis-associated interstitial lung disease. PLoS ONE. 2020;15(3): e0229997.

14. Amigues I, Ramadurai D, Swigris JJ. Current perspectives on emerging biomarkers for rheumatoid arthritis-associated interstitial lung disease. Open Access Rheumatol Res Rev. 2019;11:229-35.

15. Lee YS, Kim HC, Lee BY, Lee CK, Kim MY, Jang SJ, Lee HS, Moon J, Colby TV, Kim DS. The value of biomarkers as predictors of outcome in patients with rheumatoid arthritis-associated usual interstitial pneumonia. Sarcoidosis Vasc Diffuse Lung Dis. 2016;33(3):216-23.

16. Arnett FC, Edworthy SM, Bloch DA, McShane DJ, Fries JF, Cooper NS, Healey LA, Kaplan SR, Liang MH, Luthra HS, et al. The American Rheumatism Association 1987 revised criteria for the classification of rheumatoid arthritis. Arthritis Rheum. 1988;31(3):315-24.

17. Vitali C, Bombardieri S, Jonsson R, Moutsopoulos HM, Alexander EL, Carsons SE, Daniels TE, Fox PC, Fox RI, Kassan SS, Pillemer SR, Talal N, Weisman $\mathrm{MH}$, European Study Group on Classification Criteria for Sjogren's S. Classification criteria for Sjogren's syndrome: a revised version of the European criteria proposed by the American-European Consensus Group. Ann Rheum Dis. 2002;61(6): $554-558$.

18. Sasaki H, Kohsaka H. Current diagnosis and treatment of polymyositis and dermatomyositis. Mod Rheumatol. 2018;28(6):913-21.

19. van den Hoogen F, Khanna D, Fransen J, Johnson SR, Baron M, Tyndall A, Matucci-Cerinic M, Naden RP, Medsger TA Jr, Carreira PE, Riemekasten G, Clements PJ, Denton CP, Distler O, Allanore Y, Furst DE, Gabrielli A, Mayes MD, van Laar JM, Seibold JR, Czirjak L, Steen VD, Inanc $\mathrm{M}$, Kowal-Bielecka O, Muller-Ladner U, Valentini G, Veale DJ, Vonk MC, Walker UA, Chung L, Collier DH, Ellen Csuka M, Fessler BJ, Guiducci S, Herrick A, Hsu VM, Jimenez S, Kahaleh B, Merkel PA, Sierakowski S, Silver RM, Simms RW, Varga J, Pope JE. 2013 classification criteria for systemic sclerosis: an American college of rheumatology/European league against rheumatism collaborative initiative. Ann Rheum Dis. 2013;72(11):1747-55.

20. Raghu G, Collard HR, Egan JJ, Martinez FJ, Behr J, Brown KK, Colby TV, Cordier JF, Flaherty KR, Lasky JA, Lynch DA, Ryu JH, Swigris JJ, Wells AU, Ancochea J, Bouros D, Carvalho C, Costabel U, Ebina M, Hansell DM, Johkoh T, Kim DS, King TE Jr, Kondoh Y, Myers J, Muller NL, Nicholson AG, Richeldi L, Selman M, Dudden RF, Griss BS, Protzko SL, Schunemann HJ, Fibrosis AEJACoIP. An official ATS/ERS/JRS/ALAT statement: idiopathic pulmonary fibrosis: evidence-based guidelines for diagnosis and management. Am J Respir Crit Care Med. 2011;183(6):788-824.

21. Huuskonen O, Kivisaari L, Zitting A, Taskinen $\mathrm{K}$, Tossavainen A, Vehmas T. High-resolution computed tomography classification of lung fibrosis for patients with asbestos-related disease. Scand J Work Environ Health. 2001;27(2):106-12.

22. O'Dwyer DN, Armstrong ME, Cooke G, Dodd JD, Veale DJ, Donnelly SC. Rheumatoid arthritis (RA) 
associated interstitial lung disease (ILD). Eur J Intern Med. 2013;24(7):597-603.

23. Wang T, Zheng XJ, Ji YL, Liang ZA, Liang BM. Tumour markers in rheumatoid arthritis-associated interstitial lung disease. Clin Exp Rheumatol. 2016;34(4):587-91.

24. Xiong W, Zhao Y, Xu M, Guo J, Pudasaini B, Wu X, Liu J. The relationship between tumor markers and pulmonary embolism in lung cancer. Oncotarget. 2017;8(25):41412-21.

25. Wang W, Chen XL, Zhao SY, Xu YH, Zhang WH, Liu K, Chen XZ, Yang K, Zhang B, Chen ZX, Chen JP, Zhou ZG, Hu JK. Prognostic significance of preoperative serum CA125, CA19-9 and CEA in gastric carcinoma. Oncotarget. 2016;7(23):35423-36.

26. Maher TM, Oballa E, Simpson JK, Porte J, Habgood A, Fahy WA, Flynn A, Molyneaux PL, Braybrooke R, Divyateja H, Parfrey H, Rassl D, Russell AM, Saini G, Renzoni EA, Duggan AM, Hubbard R, Wells AU, Lukey PT, Marshall RP, Jenkins RG. An epithelial biomarker signature for idiopathic pulmonary fibrosis: an analysis from the multicentre PROFILE cohort study. Lancet Respir Med. 2017;5(12): 946-55.
27. Yamamoto S, Kobayashi S, Tanaka M, Akimoto T, Takasaki Y. Serum CA 19-9 levels in rheumatic diseases with interstitial pneumonia. Nihon Rinsho Meneki Gakkai Kaishi. 1996;19(2):128-35.

28. Strieter RM, Mehrad B. New mechanisms of pulmonary fibrosis. Chest. 2009;136(5):1364-70.

29. Satoh $H$, Kurishima K, Ishikawa $H$, Ohtsuka M. Increased levels of KL-6 and subsequent mortality in patients with interstitial lung diseases. J Intern Med. 2006;260(5):429-34.

30. Ishikawa $\mathrm{N}$, Hattori $\mathrm{N}$, Yokoyama $\mathrm{A}$, Kohno $\mathrm{N}$. Utility of KL-6/MUC1 in the clinical management of interstitial lung diseases. Respir Investig. 2012;50(1):3-13.

31. Takahashi K, Nakamura $H$, Takenouchi K, Iizawa N, Koiwa M, Sato A, Mochizuki Y, Watanabe H, Takai S. Serum KL-6 elevation and possible pulmonary involvement in patients with rheumatoid arthritis treated with biological agents. J Nippon Med Sch. 2014;81(6):364-71. 